\title{
Kenia: Detectar la sifilis durante la consulta prenatal resulta costo-efectivo
}

Frontiers in Reproductive Health

Follow this and additional works at: https://knowledgecommons.popcouncil.org/departments_sbsr-rh

Part of the Community Health and Preventive Medicine Commons, Demography, Population, and Ecology Commons, International Public Health Commons, and the Maternal and Child Health Commons How does access to this work benefit you? Let us know!

\section{Recommended Citation}

"Kenia: Detectar la sifilis durante la consulta prenatal resulta costo-efectivo," FRONTERAS Resumen de Investigación Operative. Ciudad de México: Population Council, 2001. 


\section{Kenia Infecciones del tracto reproductivo}

\author{
Investigación \\ operativa
}

Resumen 16

\section{Detectar la sífilis durante la consulta prenatal resulta costo-efectivo}

\author{
Un modelo que proporciona detección y tratamiento de sífilis en el mismo \\ establecimiento y el mismo día de la consulta prenatal, dio como resultado \\ mayores tasas de tratamiento para las clientas con prueba positiva y sus \\ parejas, a un costo razonable para el sistema de salud. La capacitación de \\ todas las enfermeras en detección y tratamiento de sífilis, así como el abasto \\ constante de los suministros esenciales para las pruebas y el tratamiento, \\ permitieron mantener los servicios activos.
}

\section{Antecedentes}

La Organización Mundial de la Salud ha estipulado que la detección y tratamiento de sífilis para todas las mujeres embarazadas resulta eficaz en función de los costos si al menos el 0.1 por ciento de ellas están infectadas. Para atender el problema de la alta tasa de sífilis entre mujeres embarazadas $(6.5-7.3 \%)$, el Concejo de la Ciudad de Nairobi (CCN) introdujo en 1989 la detección y tratamiento de sífilis materna en sus clínicas de atención prenatal. Sin embargo, su enfoque centralizado - que requiere llevar las muestras de sangre recolectadas a un laboratorio central para hacer la prueba - es ineficiente: bajo este esquema, sólo al 62 por ciento de las clientas se les hacía la prueba, y sólo el 9 por ciento de quienes obtenían resultados positivos, recibían tratamiento.

Por lo tanto, en 1992, el CCN probó un enfoque descentralizado en nueve de sus 54 clínicas de atención prenatal. Los principales aspectos del nuevo enfoque fueron: (1) aplicación in situ de la prueba rápida de reaginina en plasma (RPR, por sus siglas en inglés) por parte del personal clínico; (2) tratamiento el mismo día a las mujeres con resultado positivo por parte de las enfermeras, y (3) promoción de la notificación y presunto tratamiento a los compañeros de las mujeres con prueba positiva. Este enfoque resultó exitoso y, posteriormente, cinco clínicas adicionales lo aplicaron.

En el año 2000, el Population Council llevó a cabo un estudio de caso para analizar la efectividad, los costos y la sostenibilidad del programa descentralizado de detección de sífilis tras ocho años de operación. Se comparó la información de las estadísticas de servicio de 1998 y 1999 de 11 de las 14 clínicas del CCN con servicios de detección y tratamiento in situ, con la de siete clínicas regulares sin estos servicios. Además, se llevaron a cabo observaciones de las consultas, entrevistas de salida a las clientas, grupos focales y entrevistas a profundidad con los proveedores. También se realizaron un análisis de flujo de 1,298 pacientes y un análisis de costos.

\section{Hallazgos}

- Implementar la detección y el tratamiento de sífilis in situ dentro del programa prenatal existente resultó viable y eficaz en función de los costos. Introducir estos servicios in situ en las clínicas regulares aumenta el costo por visita en US \$1, mientras que el costo adicional de las pruebas centralizadas es de US $\$ 3.70$.

- Tanto las clínicas que proporcionan detección de sífilis in situ como las clínicas regulares alcanzaron tasas altas de tratamiento para las mujeres con prueba positiva (ver figura en la siguiente página).

- Las clínicas que contaban originalmente con los servicios in situ realizaron más pruebas de detección que las clínicas regulares. Sin embargo, no se ha podido mantener la aplicación universal de las pruebas debido a tres factores: (1) la escasez de insumos para las pruebas; (2) la rotación constante del personal capacitado, y (3) la poca supervisión del personal. 
Proporción de clientas de atención prenatal que recibieron prueba de detección y tratamiento

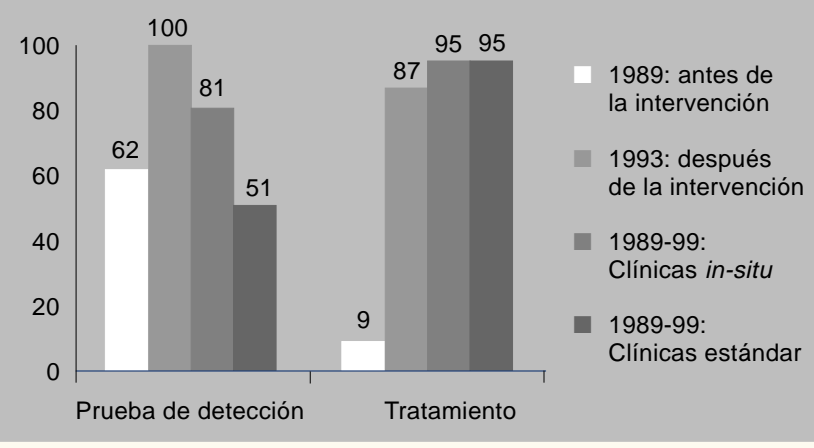

- La notificación y tratamiento a los compañeros de las mujeres con prueba positiva aumentó en las clínicas que proporcionan los servicios in situ, registrándose una tasa de retorno a la clínica para recibir tratamiento del 70 por ciento de las parejas notificadas, en comparación con el 50 por ciento registrado en 1993. La alta tasa de respuesta puede atribuirse al énfasis que el programa in situ pone en la consejería a las mujeres con pruebas positivas sobre la importancia de informar al compañero, así como al uso de una tarjeta de notificación que invita al mismo a asistir a la clínica "para recibir asesoría médica lo más pronto posible".

- En general, los centros de salud del CCN no estaban preparados para ofrecer servicios prenatales de calidad aceptable bajo ninguno de los dos modelos. En menos de la mitad de las 249 consultas observadas en las clínicas, las mujeres recibieron un examen para detectar problemas de salud general o complicaciones del embarazo, ni recibieron información esencial sobre el mismo (que abarcara señales de peligro, por ejemplo). Si bien se realizaron pruebas de sangre, rara vez se buscaron las evidencias clínicas de infección por sífilis. La mayoría de las clientas no recibieron consejería sobre la sífilis materna y otras ITS, incluyendo el VIH/SIDA. Los procedimientos para la prevención de infecciones no se cumplieron en algunos casos.
Las clientas pasaron la mayor parte de su tiempo en la clínica esperando a ser atendidas. Las clientas nuevas que no recibieron la prueba de sífilis esperaron 109 minutos y tuvieron 21 minutos de contacto con los prestadores de servicios. La prueba de detección de sífilis añadió 24 minutos al tiempo de espera y 16 minutos al tiempo de consulta. En las entrevistas de salida, tres de cada cuatro mujeres dijeron que el tiempo de espera era aceptable.

\section{Implicaciones normativas}

El hecho de no recibir tratamiento para la sífilis puede acarrear serias consecuencias para la salud de las mujeres y sus recién nacidos, entre las que se incluye un mayor riesgo de contraer VIH/SIDA, y dado el modesto costo adicional de ofrecer los servicios de detección in situ, que se calcula sea de US \$1 por visita, sería recomendable que las instituciones de salud fortalezcan y reorganicen los servicios prenatales para ofrecer servicios integrados de buena calidad que incluyan detección y tratamiento para todas las pacientes el mismo día de la consulta y en el mismo establecimiento.

También sería recomendable que las instituciones de salud proporcionen capacitación básica a todas las enfermeras para la prestación de servicios prenatales de calidad, incluyendo la detección y tratamiento in situ de la sífilis, como parte de una iniciativa más amplia para integrar la prevención y control de ITS y VIH/SIDA.

- Los centros que ofrecen servicios integrados deben contar en todo momento con los suministros y equipo médico necesarios.

Por último, se recomienda que las clínicas lleven a cabo actividades educativas grupales en la sala de espera para aprovechar mejor el tiempo de espera de las clientas.

Noviembre 2001

\section{Fuente:}

Maggwa, Baker Ndugga, Ian Askew, Elizabeth Mugwe, Bilhah Hagembe y Rick Homan. 2001. “A case study of Nairobi City Council's decentralised syphilis screening programme in antenatal clinics [Un estudio de caso del programa descentralizado de detección de sífilis en clínicas prenatales del Concejo de la Ciudad de Nairobi]," FRONTIERS Final Report. Washington, DC: Population Council.

Disponible por correo electrónico: frontiers@pcdc.org

Este proyecto se llevó a cabo con el apoyo de la AGENCIA DE LOS ESTADOS UNIDOS PARA EL DESARROLLO INTERNACIONAL (USAID) bajo el Contrato No. CCP-3030-C-3008-00. 